\title{
Similar genomic proportions of copy number variation within gray wolves and modern dog breeds inferred from whole genome sequencing
}

Aitor Serres-Armero $^{1+}\left(\mathbb{D}\right.$, Inna S. Povolotskaya ${ }^{1+}$, Javier Quilez ${ }^{1,2 \dagger}$, Oscar Ramirez ${ }^{1,3}$, Gabriel Santpere ${ }^{1,4}$, Lukas F. K. Kuderna', Jessica Hernandez-Rodriguez', Marcos Fernandez-Callejo², Daniel Gomez-Sanchez', Adam H. Freedman ${ }^{5}$, Zhenxin Fan ${ }^{6}$, John Novembre ${ }^{5}$, Arcadi Navarro ${ }^{1,2,7}$, Adam Boyko ${ }^{8}$, Robert Wayne ${ }^{5}$, Carles Vilà' ${ }^{9}$ Belen Lorente-Galdos ${ }^{1,4^{*}}$ and Tomas Marques-Bonet ${ }^{1,2,7^{*}}$

\begin{abstract}
Background: Whole genome re-sequencing data from dogs and wolves are now commonly used to study how natural and artificial selection have shaped the patterns of genetic diversity. Single nucleotide polymorphisms, microsatellites and variants in mitochondrial DNA have been interrogated for links to specific phenotypes or signals of domestication. However, copy number variation (CNV), despite its increasingly recognized importance as a contributor to phenotypic diversity, has not been extensively explored in canids.
\end{abstract}

Results: Here, we develop a new accurate probabilistic framework to create fine-scale genomic maps of segmental duplications (SDs), compare patterns of CNV across groups and investigate their role in the evolution of the domestic dog by using information from 34 canine genomes. Our analyses show that duplicated regions are enriched in genes and hence likely possess functional importance. We identify 86 loci with large CNV differences between dogs and wolves, enriched in genes responsible for sensory perception, immune response, metabolic processes, etc. In striking contrast to the observed loss of nucleotide diversity in domestic dogs following the population bottlenecks that occurred during domestication and breed creation, we find a similar proportion of CNV loci in dogs and wolves, suggesting that other dynamics are acting to particularly select for CNVs with potentially functional impacts.

Conclusions: This work is the first comparison of genome wide CNV patterns in domestic and wild canids using whole-genome sequencing data and our findings contribute to study the impact of novel kinds of genetic changes on the evolution of the domestic dog.

Keywords: Copy number variation, Dog genomics, Evolution, Domestication

\footnotetext{
* Correspondence: belen.lorente@gmail.com; tomas.marques@upf.edu

${ }^{\dagger}$ Equal contributors

${ }^{1} \mathrm{BBE}$, Institut de Biologia Evolutiva (Universitat Pompeu Fabra/CSIC), Ciencies

Experimentals i de la Salut, 08003 Barcelona, Spain

Full list of author information is available at the end of the article
} 


\section{Background}

The dog (Canis familiaris) was domesticated from the gray wolf (C. lupus) [1-4] more than 10,000 years ago, although when and where domestication happened as well as the role of humans in the process have been focus of intense debate [5-10]. Beginning several hundred years ago, modern dog breeds were established as isolated gene pools, in parallel with strong artificial selection for specific physical and behavioral phenotypes favored by humans. A large number of dog breeds have been developed since then, which has resulted in a broad variety of traits and exceptional phenotypic variation [11].

Detecting and understanding the footprint that domestication left in the canine genome is an area of active research. To this end, genetic variation in dogs and wolves has been extensively studied using single nucleotide polymorphisms (SNPs) and microsatellites [12-16]. These studies have shown that nucleotide diversity is between 1.5 and 2 times lower in dogs than in wolves as a result of a 9 to 16 -fold reduction in the effective population size associated with dog domestication $[4,17,18]$. Selective breeding further led to reduction in variation, longer linkage disequilibrium (LD) blocks and a lower number of haplotypes among purebred dogs compared to wolves and "village dogs", which have not gone through the breeding process $[15,17,19-22]$. This reduction in diversity is striking in the light of the great phenotypic variation observed in modern dog breeds [12]. Several studies have focused on the identification of functional variants responsible for phenotypic changes associated with domestication [23] or contributing to phenotypic variation of the modern dog breeds [11, 19, 24-29].

Although CNV contributes to phenotypic differences and genetic diseases [28, 30-32], structural variation in multiple canine genomes has not been thoroughly interrogated yet genome-wide. Absolute copy number $(\mathrm{CN})$ values in short genomic windows can be predicted computationally from whole genome sequencing experiments [33-40] and this approach has been used to study CNV patterns in many species. A number of studies have investigated $\mathrm{CNV}$ in dogs and wolves using experimental approaches, namely array comparative genomic hybridizations (aCGH) [30, 41-45] and intensity data from SNP genotyping arrays [46]. However, these techniques are limited to relatively low $\mathrm{CN}$ regions [47], produce $\mathrm{CN}$ values relative to the $\mathrm{CN}$ in the reference individual [48], have strong limitation in size of the detectable structural variants $[49,50]$ and only the parts of the genome in which probes have been placed can be interrogated [47].

In the present study, we aimed to investigate $\mathrm{CNV}$ regions in dogs and wolves. However, the analysis of the genomewide patterns of segregating CNV across a set of individuals is a challenging task and requires precise estimates of the absolute $\mathrm{CN}$ of each $\mathrm{CNV}$ locus for each of the individual genomes. The accuracy of all the existing methods for absolute $\mathrm{CN}$ inference decreases rapidly as $\mathrm{CN}$ increases, and thus, nearly all of the studies of $\mathrm{CNV}$ diversity up to date are limited to biallelic loci with segregating alleles $\mathrm{CN}_{1}$ and $\mathrm{CN}_{2}$ per haplotype $[40,51,52]$. In addition, methods based on read depth only produce point estimates and do not provide confidence intervals, which are extremely important to distinguish between true $\mathrm{CN}$ variability and increased technical noise (especially for higher $\mathrm{CN}$ values) [53]. This is an important caveat considering that, as reported in humans, population differentiation in loci with a high number of copies might be an important contributor to phenotypic differences $[40,54,55]$. Here, we designed a new probabilistic framework of the read depth based approach for accurate absolute $\mathrm{CN}$ inference and $\mathrm{CNV}$ detection, which enabled us to perform a comprehensive genome-wide analysis of the patterns and dynamics of CNV loci across the entire range of CNs in a set of 34 canid genomes.

\section{Results}

We analyzed a set of 34 sequenced individuals at a mean initial coverage of $16.8 \mathrm{X}[4,56,57]$. Our dataset included 12 dogs (C. familiaris), 16 gray wolves (C. lupus), 2 red wolves (C. rufus), 3 coyotes (C. latrans) and 1 golden jackal (C. aureus) (Table 1) from diverse populations and breeds across Europe, America and Asia [57].

We generated individual genome-wide fine-scale $\mathrm{CN}$ profiles using a previously published method [33]. Further, we developed and applied a new probabilistic approach, which allowed us to overcome some of the limitations of the previous methods by estimating probabilities for each $\mathrm{CN}$ and broaden the analysis to include loci of high $\mathrm{CN}$.

\section{Validation}

We validated our computational predictions with the available aCGH data [43] for 14 of the samples that are common in both studies (Table 1). We compared "digital" $\log 2$ ratios between the reference individual ("bxr") and each of the other samples included in the aCGH study [43], which showed a high correlation with the aCGH log2ratios (mean correlation coefficient $R=0.77 \pm 0.06$, Additional file 1: Table S1). Additionally, $95.4 \pm 3.3 \%$ of windows with sample specific $\mathrm{CN}$ gains relative to the reference individual (Boxer) have passed the validation threshold (See METHODS and Additional file 1: Table S1). Boxer specific duplications had a lower validation rate $(69.3 \pm 6.8 \%)$, most likely as a result of sequencing biases specific to this sample (Additional file 1: Figure S1).

\section{Genomic duplications}

Duplicated genomic regions spanned 114.05 Mbps (43.44 Mbps in autosomal chromosomes and 70.69 Mbps in unplaced scaffolds) or about $5 \%$ of the size of dog autosomes. Dogs have 111.82 Mbps of duplicated 
Table 1 Samples and sequencing coverage

\begin{tabular}{|c|c|c|c|c|c|c|c|c|}
\hline Species & Sample & Abbreviation & HMM function & Raw coverage & Effective coverage & aCGH data & Dataset & Diversity analysis \\
\hline Dog & $\begin{array}{l}\text { Chinese indigenous } \\
\text { dog }\end{array}$ & $\operatorname{Dog} C 12$ & Training & 9.83 & - & No & Wang et al. & No \\
\hline Dog & Dingo & $\operatorname{din}$ & Analysis & 7.09 & 5.1 & No & Freedman et al. & Yes \\
\hline Dog & Basenji & $\mathrm{mba}$ & Analysis & 11.8 & 8.49 & Yes & Freedman et al. & Yes \\
\hline Dog & Kerry Blue Terrier & ali & Analysis & 21.28 & 15.32 & No & Fan et al. & Yes \\
\hline Dog & Boxer & bxr & Analysis & 31.27 & 22.29 & No & Fan et al. & Yes \\
\hline Dog & English cocker & cec & Analysis & 11.81 & 8.5 & No & Fan et al. & Yes \\
\hline Dog & Labrador retriever & dlr & Analysis & 12.6 & 9.07 & No & Fan et al. & Yes \\
\hline Dog & Chinese crest & $\mathrm{jcc}$ & Analysis & 19.04 & 13.71 & No & Fan et al. & Yes \\
\hline Dog & Standard poodle & osp & Analysis & 12.91 & 9.29 & No & Fan et al. & Yes \\
\hline Dog & Belgium Malanois & DogBM & Analysis & 10.11 & 7.57 & No & Wang et al. & Yes \\
\hline Dog & German shepherd & DogGS & Analysis & 9.56 & 5.61 & No & Wang et al. & Yes \\
\hline Dog & Tibetan Mastiff & DogTM & Analysis & 10.37 & 5.8 & No & Wang et al. & Yes \\
\hline Gray wolf & Wolf Russia & GW3 & Training & 11.1 & - & No & Wang et al. & No \\
\hline Gray wolf & Wolf China & chw & Analysis & 17.94 & 12.91 & Yes & Freedman et al. & Yes \\
\hline Gray wolf & Wolf Croatia & $\mathrm{crw}$ & Analysis & 9.73 & 6.94 & No & Freedman et al. & Yes \\
\hline Gray wolf & Israeli wolf & isw & Analysis & 7.37 & 5.26 & No & Freedman et al. & Yes \\
\hline Gray wolf & Wolf Great Lakes & glw & Analysis & 26.8 & 19.3 & Yes & Fan et al. & Yes \\
\hline Gray wolf & Wolf India & inw & Analysis & 27.42 & 19.74 & Yes & Fan et al. & Yes \\
\hline Gray wolf & Wolf Iran & irw & Analysis & 30.15 & 21.71 & Yes & Fan et al. & Yes \\
\hline Gray wolf & Wolf Italy & ita & Analysis & 7.59 & 6.07 & Yes & Fan et al. & Yes \\
\hline Gray wolf & Wolf Mexico & mxa & Analysis & 25.64 & 18.46 & Yes & Fan et al. & Yes \\
\hline Gray wolf & Wolf Mexico & $m \times b$ & Analysis & 7.08 & 5.66 & No & Fan et al. & No \\
\hline Gray wolf & Wolf Portugal & ptw & Analysis & 28.46 & 20.49 & Yes & Fan et al. & Yes \\
\hline Gray wolf & Wolf Spain & spw & Analysis & 28.88 & 20.79 & Yes & Fan et al. & Yes \\
\hline Gray wolf & Wolf Yellowstone & ysa & Analysis & 28.21 & 20.31 & Yes & Fan et al. & Yes \\
\hline Gray wolf & Wolf Yellowstone & ysb & Analysis & 18.82 & 13.55 & Yes & Fan et al. & No \\
\hline Gray wolf & Wolf Yellowstone & ysc & Analysis & 8.44 & 6.75 & Yes & Fan et al. & No \\
\hline Gray wolf & Wolf China & GW4 & Analysis & 9.61 & 6.75 & No & Wang et al. & No \\
\hline Coyote & Coyote California & $\mathrm{cac}$ & Training & 26.87 & 19.35 & No & Fan et al. & No \\
\hline Coyote & Coyote Alabama & alc & Analysis & 7.69 & 5.54 & No & Fan et al. & No \\
\hline Coyote & Coyote Midwest & mwc & Analysis & 9.11 & 6.56 & No & Fan et al. & No \\
\hline Jackal & Golden Jackal Kenya & jaa & Analysis & 27.47 & 19.78 & Yes & Freedman et al. & No \\
\hline Red wolf & Red wolf & rwa & Analysis & 30.28 & 21.8 & No & Fan et al. & No \\
\hline Red wolf & Red wolf & rwb & Analysis & 7.72 & 6.17 & No & Fan et al. & No \\
\hline
\end{tabular}

Sequences were retrieved from previously published work from Fan et al. [57], Freedman et al. [4] and Wang et al. [56]. The raw coverage is calculated from the total number of reads before mapping and referred to the 2,413,045,422 bps of the prepared version of CanFam3.1. The effective coverage is calculated after removing poor-quality sequencing lanes and read ends. For 14 samples aCGH data from Ramirez et al. [43] were available. Coyote, jackal and red wolf samples were combined as a single group for the analyses

sequence, gray wolves $111.46 \mathrm{Mbps}$ and related canids 109.74 Mbps. We found, that $79 \%$ of the genomic duplications were present in all the individuals $(89.72$ Mbps in total, 24.03 Mbps in chromosomes and 65.70 Mbps in unplaced scaffolds), 93.04 Mbps ( 83\%) were present in all the dogs and $95.53 \mathrm{Mbp}(\sim 86 \%)$ in all the wolves (Fig. 1a). Dogs and gray wolves showed the same average amount of duplicated sequence per individual (104.21 \pm 1.89 and $105.54 \pm 0.71$ Mbps, respectively, Fig. 1b) and $38.22 \mathrm{Mbps}$ were duplicated in at least one individual from each subspecies excluding unassembled scaffolds (Fig. 1c). The average length of duplicated segments did not depend on the sample coverage (Additional file 1: Figure S2). 


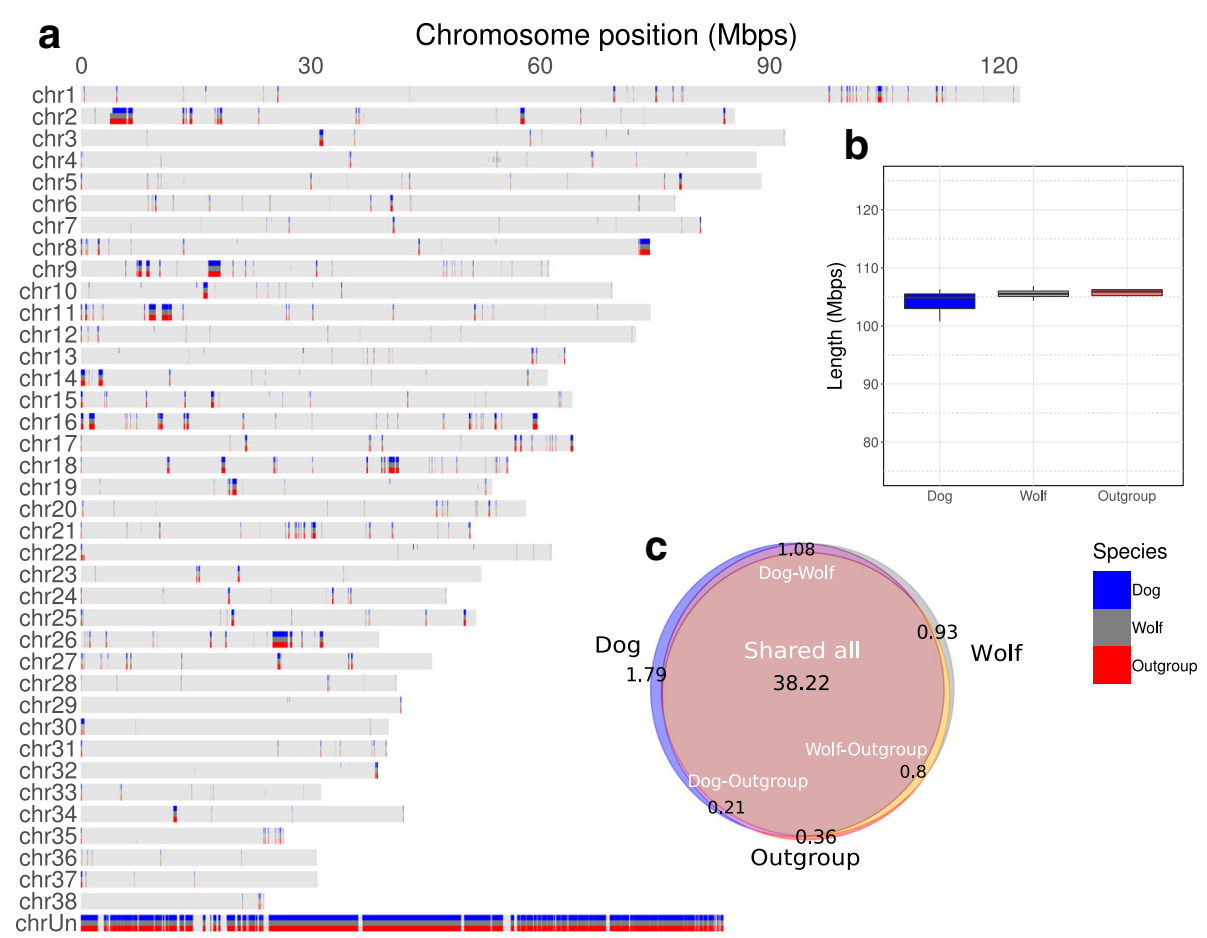

Fig. 1 Landscape of canine segmental duplications. a Genome-wide map of canine SDs. Autosomes are represented by horizontal bars, and each mark represent a duplicated region identified in at least one sample of the group indicated. $\mathbf{b}$ Total length of genomic duplications identified per subspecies. c Venn diagram showing intersection of duplicated regions identified in dogs, gray wolves and canines in the outgroup (chrUn excluded)

We found that the set of genomic duplications detected in the 34 canine samples overlapped with 433 genes annotated in the CanFam3.1 dog genome assembly, an overlap significantly higher than the random expectation (randomization $p$-value $=0.0023$ ) (Additional file 1: Figure S3). Moreover, we found a significant enrichment of duplicated genes involved in detection of chemical stimulus and G-protein coupled receptor signaling pathways, both with $\mathrm{p}$-val $<10^{-30}$ (Additional file 1: Table S2). These two pathways are closely associated with the perception and transduction of smell and other sensory functions. We also detected a significant enrichment in the pathways of immunoglobulin production and phagocytosis recognition with a $p$-value of $\sim 10^{-6}$. Many essential genes were duplicated in all of our samples, including major cytoskeleton components, a number of ribosomal genes/proteins, mitochondrion maintenance and ubiquitination enzymes or DNA repair mechanisms among many others.

We further looked at the private duplications, present in one subspecies and not in the other. We restricted subsequent analysis to include only 11 dogs and 11 wolves from distinct populations (Table 1, Additional file 1: Table S3), consequently these differences do not result from different sample sizes of dogs and gray wolves (see METHODS). The number of duplications that were unique to dogs (3.67 Mbps or $23.29 \%$ of dog duplications) was substantially greater than for gray wolves (2.19 Mbps or $\sim 1.97 \%$ of gray wolf duplications) and they mainly corresponded to events in single individuals (Additional file 1: Figure S4) and none of the private duplications was shared by more than 7 individuals. These private duplications were also significantly enriched in genes for both dogs (randomization $p$-value $=0.0075$ ) and wolves (randomization $p$-value $=0.003$ ) with genes involved in iron homeostasis and elastin catabolism overrepresented in dogs, and genes involved in arginine transport overrepresented in wolves (Additional file 1: Table S4).

\section{Genomic proportion of CNV}

Our CN calls allowed us to identify windows with segregating $\mathrm{CN}$ alleles within populations. We assessed whether the proportion of the genome classified as $\mathrm{CNV}$ was reduced in the dog lineage relative to the gray wolf, as has happened for nucleotide diversity (Fig. 2a) reflecting domestication and breed creation bottlenecks. As an overall measure of the fraction of the genome with segregating $\mathrm{CNV}$ in either subspecies, we used the number of 1 -Kbps windows for which at least two individuals presented non-overlapping $\mathrm{CN}$ intervals (further referred to as variable windows specifically or $\mathrm{CN}$ variability globally) divided by the total number of 1 -Kbp windows called inside duplications, taken as the most likely substrate for CNVs $[58,59]$. In striking contrast to the 1.6-fold reduction in single nucleotide diversity in our dataset of dogs (in accordance with estimates of 1.5 to 2fold reduction reported previously $[4,17,18]$, see Additional 

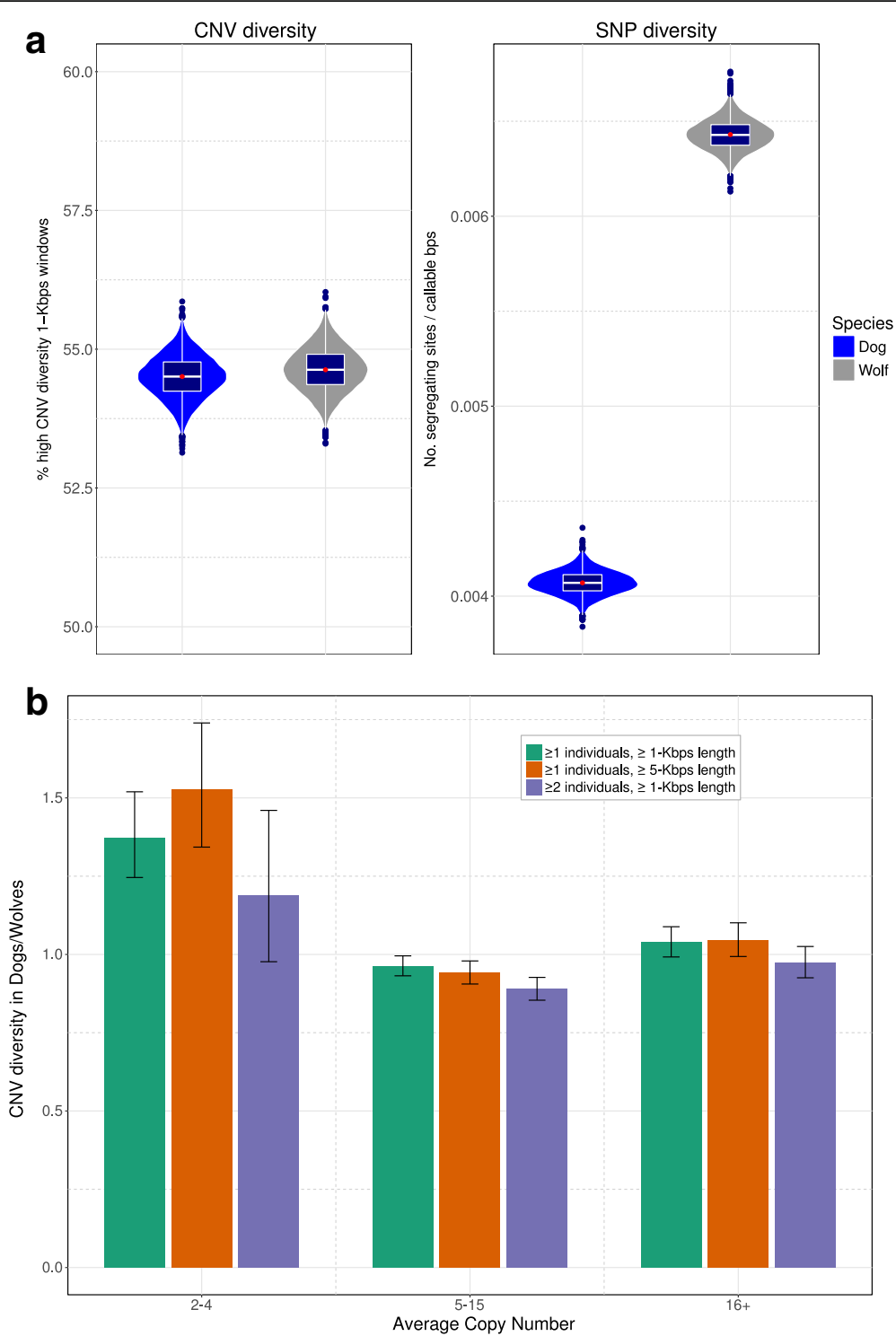

Fig. 2 Proportion of genomic CNV and SNP diversity in dogs and gray wolves. a Boxplots indicate the observed values of overall genomic proportion of CNVs and SNP diversity in dogs and gray wolves. Violin plots correspond to 5000 bootstrap values. $\mathbf{b}$ Ratios of the level of CNVs in dogs to the level of CNVs in wolves for low (2-4), medium (5-15) and high (16+) copy number categories. In green all the regions are taken into account, in orange short regions (less than 5 consecutive windows) of variable $\mathrm{CN}$ are filtered and in purple singletons are filtered

file 1: Tables S4 and S5), we found similar proportion of the duplicated genome space with CNVs in the two canine subspecies $(54.5 \%$ and $54.6 \%$ variable windows per total number of duplicated windows in dogs and wolves respectively) (Fig. 2a, Additional file 1: Table S6). Among all variable windows in dogs, $78.8 \%$ are also variable in wolves, while for wolves this proportion is slightly higher (80.9\%). Most of these regions represent in principle, variability originated before the lineage split, whereas those regions not shared (21.2\% and $19.1 \%$ respectively), represent subspeciesspecific variability, which could potentially contribute to functional differences between the two subspecies (Additional file 1: Figure S5). Alternatively, these regions may represent independent inheritance of $\mathrm{CNVs}$ from a common ancestor.

We sought then to eliminate the possibility that artifacts of our $\mathrm{CN}$ calling algorithm might influence our estimates. Given the known differences in accuracy of depth of coverage methods for different $\mathrm{CN}$ magnitudes, we first divided all genomic duplications into three categories according to their corresponding $\mathrm{CN}$. Specifically, these categories included duplications of low $\mathrm{CN}$ (mean $\mathrm{CN}$ across all windows in all duplicated individuals between 2 and 4), medium $\mathrm{CN}$ (mean $\mathrm{CN}$ between 4 and 15) and high $\mathrm{CN}$ (mean $\mathrm{CN}$ larger than 15). We then calculated variability levels for each of the categories separately (Additional file 1: 
Table S6). Surprisingly, the proportion of CNV windows within genomic duplications with low $\mathrm{CN}(2-4)$, is even higher in dogs than in wolves ( $28 \%$ and $20 \%$ respectively). In this category we assessed the quality of the calls of variable windows for each pair of samples with a two-way aCGH comparison. We required that the absolute value: $\log _{2} \frac{C N_{\text {Sample } 1}}{C N_{\text {Sample } 2}}=a C G H_{\text {Sample } 1}-a C G H_{\text {Sample } 2}$ exceeds the cutoff of $a C G H_{\text {CUTOFF }}= \pm 3 * \sigma_{a C G H}(C R)$ (see METHODS for details) for all the windows with predicted $\mathrm{CN}$ differences between the two samples, when one of the samples was not predicted to be duplicated. We thus validated $89 \%$ of windows per sample for relative losses and $88 \%$ per sample for relative gains (median values, Additional file 1: Table S7).

To further investigate if our measure of $\mathrm{CN}$ variability is affected by singletons, we repeated the analysis requiring a minimum of two individuals to be called with a different CN. Even so, dogs and wolves presented similar genomic proportions of CNVs and the value in the low $\mathrm{CN}$ category is still slightly higher for dogs (Fig. 2b, Additional file 1: Table S6). Finally, we tested whether the similar levels of genomic variation are not driven by hyper variable duplication breakpoints [60] and are not a result of inaccurate calls of short variable regions. To do so we required for $\mathrm{CN}$ regions to be comprised of a minimum of 5 consecutive windows which are identified as variant within the population, and still found overall similar genomic proportion of CNVs comparing dogs and wolves (Fig. 2b, Additional file 1: Table S6).

Variable duplicated genomic segments, defined as 1Kbps windows for which there were at least two individuals with non-overlapping $\mathrm{CN}$ intervals, are enriched in genes in the low and medium $\mathrm{CN}$ categories for both lineages (dogs: $\mathrm{p}_{\mathrm{CN} 2-4}=0.018$ and $\mathrm{p}_{\mathrm{CN} 5-15}=0.023$; wolves: and $\mathrm{p}_{\mathrm{CN} 2-4}=0.014$ and $\left.\mathrm{p}_{\mathrm{CN} 5-15}=0.053\right)$ and many of these genes are involved in both innate immunity (6 genes related to phagocytosis recognition) and adaptive immunity (15 genes involved in immunoglobulin production and $\mathrm{MHC}$ maturation). A striking enrichment was found in the pathway of DNA recombination and the most significant signal belonged again to olfactory receptor activity (Additional file 1: Table S8).

We further looked for genes which show a high degree of $\mathrm{CN}$ differentiation between the two subspecies based on the $\mathrm{V}_{\mathrm{ST}}$ statistic. We recover a number of genic CNVs previously reported to be associated with the dog specific phenotypes. Among these genes is the paralogue to the canine alpha-2B-amylase gene $(A M Y 2 B)$, which catalyzes the first step in the digestion of dietary starch and glycogen (Fig. 3a and Additional file 1: Table S9). Another case of CN expansion in dogs is a $150-$ Kbps duplication in chromosome 24 [16, 42]. This duplication spans three members of the signal-regulatory protein (SIRP) gene family, which mediate immune-cell regulation
[61] (Fig. 3b and Additional file 1: Table S9). Similarly, the CBR1 gene (Fig. 3c), coding for a carbonyl reductase enzyme involved in the degradation of both environmental and biologically synthesized quinones, lies within a region duplicated in most samples with some dog samples having a higher number of copies (Additional file 1: Table S9).

\section{Discussion}

Inferring absolute $\mathrm{CN}$ values from sequencing read depth and determining gains in the number of copies is not trivial. Among computational methods, read depth based approaches are the most accurate [62]. Here, we develop an accurate probabilistic expansion of the sequencing read depth based method to call $\mathrm{CN}$ genome wide and use this method to produce fine-scale maps of genomic duplications and CNV regions in dogs, gray wolves and the more basal coyote and golden jackal lineages. The novelty of our approach relies in the population-wide Bayesian probabilistic method to $\mathrm{CN}$ estimation, hence allowing us to reliably compare $\mathrm{CN}$ values across groups of genomes.

The CNV and duplication maps that we present in this study greatly improve on the landscape of structural variation in the canine genome. We analyzed a set of dogs from different breeds, but we additionally included a wide range of gray wolf samples from a broad geographic distribution and several individuals from other wild canine species. All samples were previously sequenced $[4,56,57]$ by next generation sequencing and we utilized a computational read depth approach to estimate fine-scale $\mathrm{CN}$ for each individual.

The main objective of our study was to investigate whether the proportion of the genome with $\mathrm{CNV}$ regions is reduced in dog compared to gray wolf genomes, which would indicate a reduction of $\mathrm{CN}$ polymorphisms similar to the expectations based on SNP diversity and the inferred bottlenecks. To do that, precise estimates of the absolute $\mathrm{CN}$ of each $\mathrm{CNV}$ locus for each of the individual genomes and a probability associated to them are required. We applied an HMM prediction, local multi-sample regenotyping and created accurate interval estimates of absolute CNs. We further validated our calling method with an available experimental dataset and found that the accuracy of the method is comparable and in some cases slightly superior to the accuracy of previous methods for copy gain predictions. Accuracy of the calls varies across samples but is not dependent on coverage depth (Additional file 1: Figure S6), as expected since the uncertainties associated with coverage are taken into account by HMM predictions and multi-sample re-genotyping.

Our fine-scale duplication maps indicate that dog genomes present similar genomic proportion of CNV compared to those of gray wolves (Fig. 2a). Nucleotide diversity in dogs compared to their canine ancestors has 

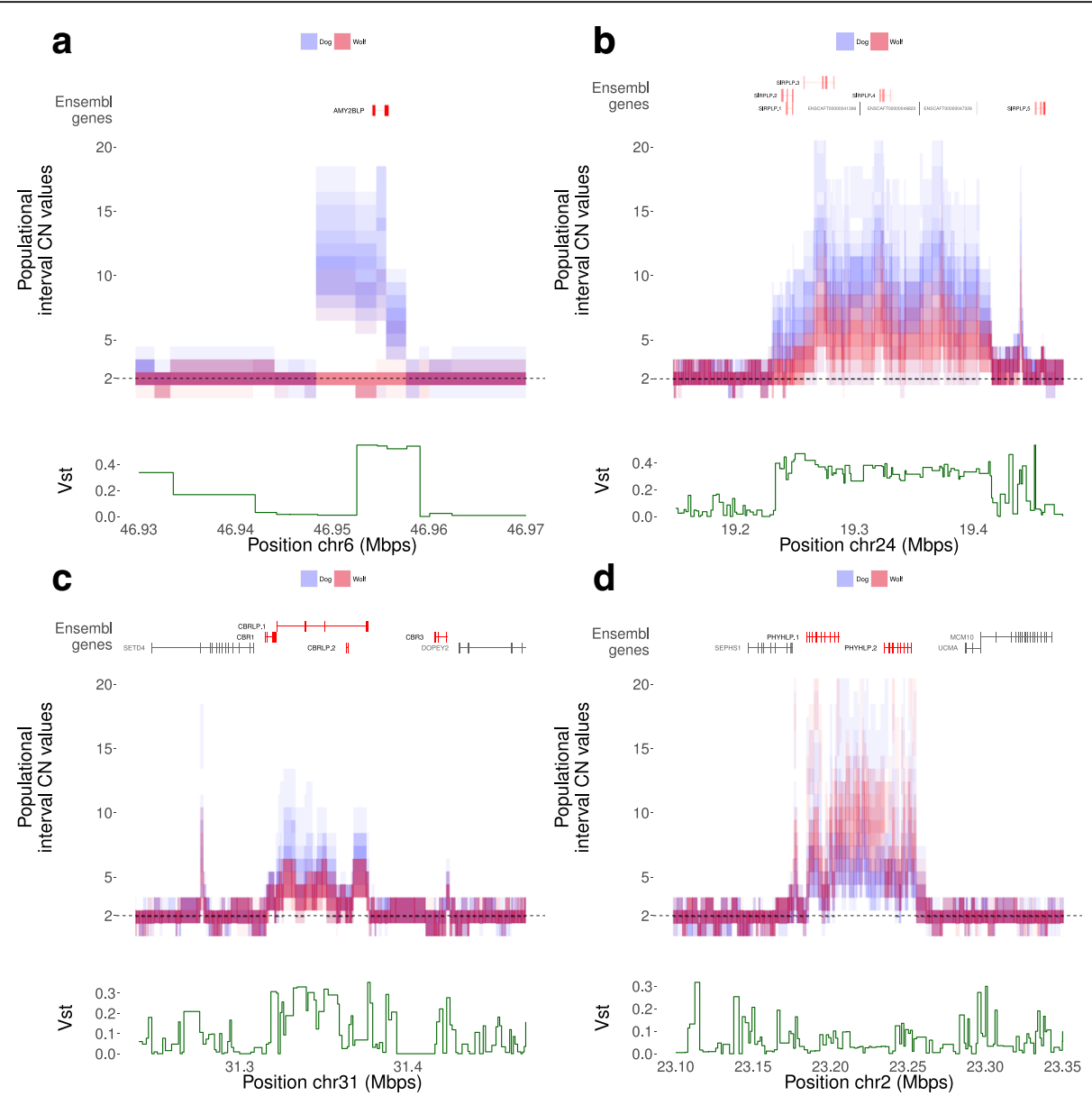

Fig. 3 Genes in high $V_{S T}$ between dogs and wolves. (a) AMY2B (b) SIRP (c) CBR1 (d) PHYH

been reduced genome-wide, as reported previously [4, $17,18]$ and supported by our data in an extended set of samples (Fig. 2a, Additional file 1: Table S5 and Additional file 1: Table S6). This reduction in nucleotide diversity has been attributed to the population bottlenecks and inbreeding that dogs have suffered as a result of domestication and the creation of dog breeds [13, 20, 63]. With regard to duplications, we observe that $\sim 80 \%$ of the CNV sites are mostly shared by both subspecies. It is notable that still $\sim 20 \%$ of these genomic CNV regions are not shared, and they might then contribute to the phenotypic plasticity observed in modern dog breeds or represent different sampling of $\mathrm{CNV}$ regions from a common ancestor.

We explicitly addressed potential biases that could affect the calculation of the proportion of CNVs. First, the proportions are maintained when considering a high confidence subset of regions, for which at least two individuals are called with a different $\mathrm{CN}$. As for each individual we require the cumulative probability of the $\mathrm{CN}$ interval to reach at least 0.99 , the probability that two distinct individuals would be called incorrectly is lower than $10^{-4}$. Second, accuracy of duplication calls increases with the length of the duplicated region [33] and the same is true with the accuracy for the calls of variability. After exclusion of all short variable segments, the resemblance between $\mathrm{CN}$ variation levels in the two subspecies is still maintained and the relative genomic $\mathrm{CNV}$ proportion in the low $\mathrm{CN}$ category even increases in dogs (Fig. 2b).

Finally, perhaps the greatest challenge in our estimates of the genomic proportion of variable duplications, is the fact that the total length of duplications represented in the dog assembly that are unique to dog samples might be either collapsed or misrepresented. To determine the extent of this problem in our estimates, we used the duplications identified in the genome with the whole-genome assembly comparison (WGAC) [42] to count each duplication detected in each subspecies. After correcting for duplications annotated in CanFam2, we found a slight increase in the proportion of CNVs observed in the dog compared to the wolf, although the final magnitudes were reduced $15-20 \%$ in both 
subspecies (Additional file 1: Figure S7). It is worth mentioning that our approach is based on counting the proportion of variable windows or equivalently the proportion of total length of variable duplications in the entire duplicated space and therefore it is just an estimation of the actual duplication units. For a more accurate assessment, better resolution of duplication events and breakpoints is required, which could be achieved by whole genome reconstruction based on long read sequencing technologies.

Altogether, similar levels of $\mathrm{CNV}$ load in dogs and wolves are extremely unlikely to be explained by an artifact or a bias alone. A key question is then why $\mathrm{CN}$ variability is not as reduced in dogs compared to single-nucleotide variation. Below, we considered each of the forces driving mutation-selection-migration equilibrium separately.

There are two scenarios in which selection might increase CNV levels in dogs above those expected given their demographic history. First, the maintenance of relatively high CNV levels in dogs is consistent with diversifying selection among different canine populations if regions of $\mathrm{CNV}$ are strongly functional. However, if that is the case, selected functional variants should show high frequency in breeds sharing a trait under selection and be at low frequency or absent in other dogs, resulting in a high overall proportion of genomic $\mathrm{CNV}$. Although, this idea is difficult to test with the current dataset due to the limited number of samples per dog breed, data with aCGH suggest that most of the CNV found in dogs are not shared within breeds but across individuals of different breeds [30]. However, this data does not eliminate across breed variability in high CNs, which would not be detected given the lower dynamic range for such values in aCGH. Alternatively, domestication has relaxed selective pressure on dogs [64] and the consequences of this relaxation can be seen in differences in coding sequence variation [65]. Then, if CNV is generally slightly deleterious, the reduced efficiency of natural selection in small populations during the domestication bottleneck might affect CNVs differently than general SNP diversity especially if the distribution of selective effects is biased toward a greater frequency of neutral or nearly neutral variants in CNVs.

The CNV mutational landscape might also be altered in the canine lineage. Notably, the recombination hotspot gene PRDM9 gene was pseudogenized in the dog genome. This gene is involved in recombination and novel $\mathrm{CNV}$ formation in primate and rodent lineages $[66,67]$. Its absence in the dog genome might imply different conditions for $\mathrm{CNV}$ formation in the canine lineage. The genomes of closely related domestic cat, panda and ferret all carry a functional copy of PRDM9. Interestingly, a region with $R P A 3$, one of the genes which binds and stabilizes single-stranded DNA during DNA replication and plays a role in double-strand break repair via homologous recombination, is duplicated in all canid genomes in our study and is variable in dogs. Given $80 \%$ of CNVs are shared between the two subspecies, many of them likely originated before the two lineages split, but it could also indicate recurrent duplication events happening at hotspots. However, great uncertainty exists about the overall mutation rate of SNPs [22,68] and CNV in canines and even less is known about the variation of this rate between dogs and wild canids.

Finally, a reduction in our estimates of CNV relative to SNP diversity also could have been accomplished by reducing the number of genotypes $[69,70]$ that are segregating in dogs. $\mathrm{CNV}$ loci carry on average more alleles than SNP loci, which normally carry just two [71-73]. Although the dynamics of the loss of the number of alleles might be similar between two types of variation, the levels of variability in case of $\mathrm{CN}$ will be affected less $[35,73]$. The number of alleles per loci is higher for high $\mathrm{CN}$ regions [72] and thus, even with a significant reduction of the number of alleles per locus, the level of variability of those high $\mathrm{CN}$ loci will not be reduced to the same degree. This effect might underlie the dynamics of our median and high CN categories. Remarkably, duplications with relatively low mean $\mathrm{CN}$ are consistently more variable in dogs than in wolves. These low $\mathrm{CN} \mathrm{du}$ plications are significantly enriched in genes and some have subspecies specific variants, suggesting to a certain extent they might be novel and contribute to functional changes that have occurred after the lineages split.

Regardless of the proposed scenarios, some of the CNV loci with a high degree of variability in dogs or wolves, and specifically gene expansions in $\mathrm{CN}$ in the dog lineage, might affect phenotypic differences between subspecies given that $20 \%$ are unique to one of these subspecies. A good example is the unique amplification of the amylase gene $\mathrm{CN}$ in all dogs, as opposed to the single-copy number in almost all gray wolves, which has been linked to a starch-rich diet in dogs [23] (Fig. 3a). Another example is a highly variable tandem duplication of the $P H Y H$ gene [42], which in humans is linked to Refsum disease, with multiple epiphyseal dysplasia among variable features [74] (Fig. 3d). In addition, homozygous $P H Y H$ knockout mice exhibit slightly reduced tibia length [75]. We also detected a remarkable enrichment in the levels of SDs and CNVs in the pathways of immunoglobulin production and phagocytosis recognition, as a $\mathrm{CNV}$ region comprising the cluster of SIRP genes (Fig. $3 \mathrm{~b}$ ), which are involved in the adaptive immune system [61]. The levels of immunoglobulin A have been shown to vary greatly across dog breeds [76] but, to our knowledge, copy number has never been 
studied as a possible cause for this variation. An example of natural and artificial selection acting in opposite directions might be the widespread duplication upstream of the KITLG gene, which is linked to the increased risk for squamous cell carcinoma in black standard poodles [77]. KITLG locus has been shown to be under strong selective pressure in dogs [19] and a number of other species [78-80]. Interestingly, in humans and stickleback fish this locus is associated with variation in skin pigmentation [81, 82] and therefore possibly also plays a role in coat color and patterning in dogs. Thus, high frequency of this duplication might be explained by artificial selection favoring coat color traits preferred by humans, despite its negative impact on overall fitness.

\section{Conclusions}

We present the first genome-wide assessment of $\mathrm{CNV}$ landscape in canids based on $\mathrm{CN}$ maps generated from high-coverage whole genome sequencing data. The novelty of this study resides in its focus on structural genome variation, which has not been as extensively explored as single-nucleotide variation in canids $[4,17$, 19-22]. Additionally, we present a novel method for the application to the whole-genome sequencing read depth data to predict absolute genomic $\mathrm{CN}$ under a probabilistic framework. We find that the proportion of genomewide CNVs in dogs and wolves has been maintained at similar levels in contrast to the decline of nucleotide variation seen in dogs. This result could reflect diversifying selection among dog breeds and populations if $\mathrm{CNV}$ are generally functional as with $A M Y 2 B$ [43]. The enrichment of genes in $\mathrm{CNV}$ regions further supports this assertion. Furthermore, we identify genes with divergent $\mathrm{CN}$ variation in dogs and gray wolves, which might have contributed to phenotypic and behavioral differences between the two subspecies. Determining the functional importance of $\mathrm{CNV}$ and amount of dog breed specific CNVs should be a focus of future studies.

\section{Methods}

\section{Samples and sequencing data}

We use sequence data from a panel of 22 canids including 6 dogs, 13 wolves and 3 coyotes sequenced previously [57]. Further, we included the genomes for another 12 canids recently published $[4,56]$, provided that they had a raw coverage greater than $5 \mathrm{X}$ (see below). Altogether, our final dataset comprised 12 dogs, 16 gray wolves, 2 red wolves, 3 coyotes and 1 golden jackal (Table 1) at a mean initial coverage of $16.8 \mathrm{X}[4,56,57]$. Each dog sample was from a different so-called modern dog breed with the exception of the Dingo, Basenji and Chinese indigenous dog, which are typically regarded as old lineages. The wolves were sampled from a broad geographic distribution and included a family trio (male, female and offspring) from Yellowstone. For the subsequent analyses we considered the red wolves, coyotes and the jackal samples as a single group (referred to as "outgroup").

\section{Pipeline for calling copy number from sequencing data}

We extended a read depth based approach for detection of SDs with a HMM for $\mathrm{CN}$ prediction from raw sequencing read depth and incorporated it to the pipeline for calling $\mathrm{CN}$ and $\mathrm{CNV}$ regions genome-wide (see Fig. 4 for the pipeline overview). To create raw, continuous, genome-wide CN predictions we applied a previously described [33] approach, which consists of the following steps:

(i) Masking of over-represented kmers in the assembly. In addition to the repeats already masked in the UCSC Genome Browser [83] version of CanFam3.1 with RepeatMasker [84] and Tandem Repeat Finder [85], we sought to identify and mask potential hidden repeats in CanFam3.1. In order to do so, chromosomes were partitioned into 36-bps kmers (with adjacent kmers overlapping $5 \mathrm{bps}$ ) and the resulting kmers were mapped against CanFam3.1 using mrsFast [86]. Then we masked positions in the assembly mapped by kmers with more than 20 placements in the genome, resulting in $6,910,707$ bps additionally masked compared to the original CanFam3.1.

(ii) Mapping 36-bps reads against the assembly. Illumina reads from each individual were split into 36-bps portions (positions $10-45$ and $46-81$ of the original reads in order to exclude the lower-quality ends of the reads) and mapped to the prepared version of CanFam3.1 using mrFast [86].

(iii) Read depth calculation in 1-Kbps non-overlapping windows of non-repetitive sequence. To avoid edge problems with masked regions, which would underestimate the $\mathrm{CN}$, the 36 bps flanking the masked regions were masked as well (referred to as 36-bps padding onwards). We then calculated the read depth in 1 -Kbps non-overlapping windows of non-masked sequence.

(iv) $\mathrm{GC}$-corrected absolute $\mathrm{CN}$ estimation from read depth. Read depth values in 1-Kbps windows were then corrected for GC bias using a set of diploid control regions. Control regions were defined as a set of diploid windows totally included in autosomal regions that had not been reported as a CNV in previous studies [4, 41, 42, 87]. These studies were based on CanFam2 and we lifted over the final set of control regions to CanFam3.1. Finally, we removed gaps (plus a 36-bps padding at the start and end of the gap) from the control regions in CanFam3.1. Altogether, this resulted in 21,260 control regions (mean $=94.9 \mathrm{Kbps}$ ) with a total size of 2,017,239,131 bps (83.7\% of CanFam3.1) and the majority being smaller than $500 \mathrm{Kbps}$. Of the total 1,151,822 1-Kbps windows 998,077 (86.7\%) and 153,745 (13.3\%) were control and non-control, respectively. 


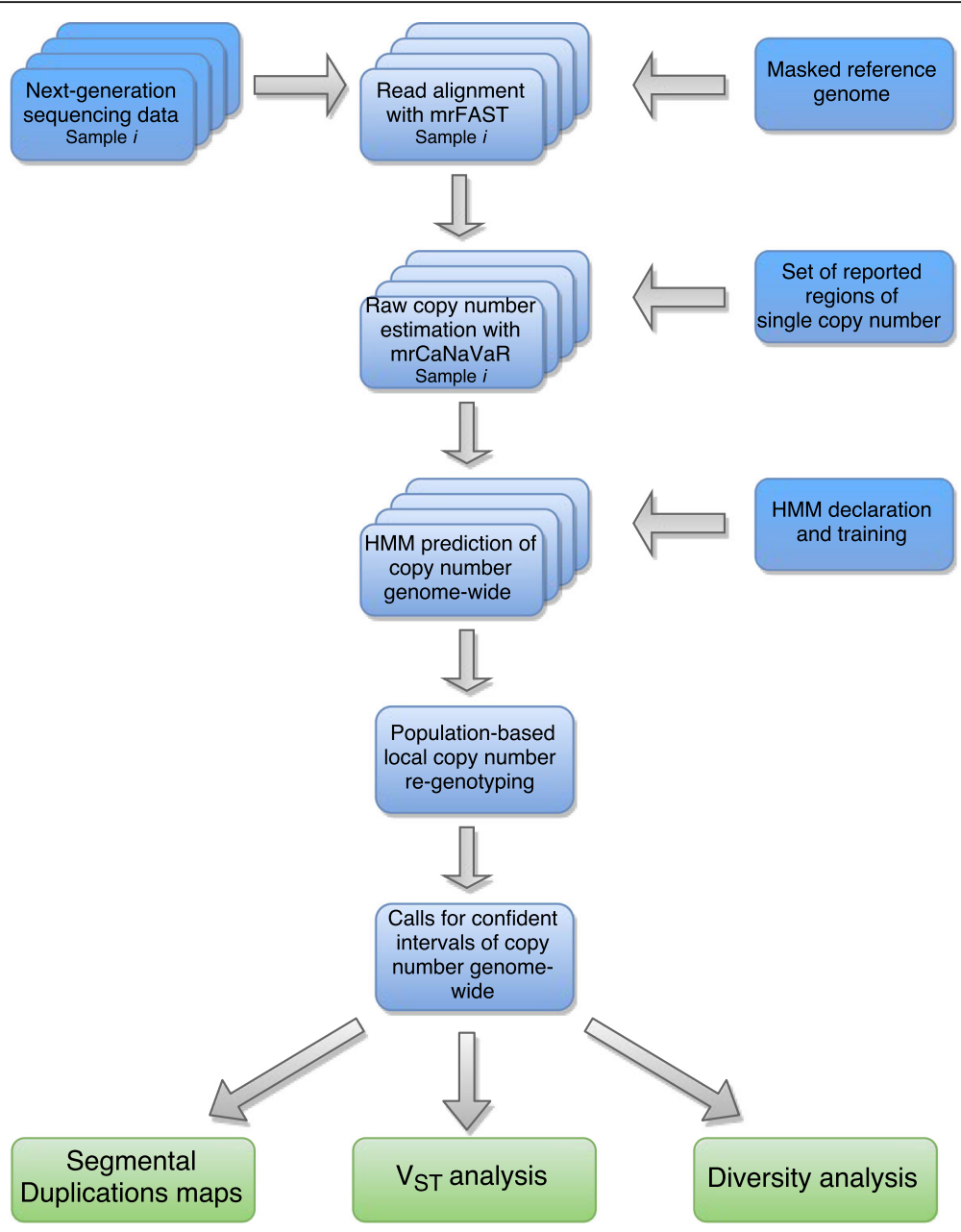

Fig. 4 Pipeline for absolute copy number calling

(v) Raw $\mathrm{CN}$ estimation. Finally, we determined $\mathrm{CN}$ in the 1-Kbps windows of non-repetitive sequence as the read depth of each window divided the mean read depth in the control regions set.

We noted that this setup is equivalent to a Hidden Markov Process, where hidden states correspond to the true integral $\mathrm{CN}$ of the genomic region, emission symbols correspond to $\mathrm{CN}$ estimates based on read depth, emission probabilities are drawn from the corresponding read depth distributions and transitions between states correspond to genomic changes of the $\mathrm{CN}$ in the adjunct genomic regions. We thus applied this HMM approach to estimate the probabilities of each $\mathrm{CN}$ for $1 \mathrm{Kbp}$ windows. We declared $\mathrm{HMM}$ states as a set of all possible $\mathrm{CN}$ for low $\mathrm{CN}$ (from $C N=0$ to $C N=20$ ) and their corresponding emission distributions as normal distributions with the mean corresponding to the $\mathrm{CN}, \mu_{\mathrm{N}}=N$, and standard deviation derived from standard deviation in control regions $(C R$, $C N=2)$ as $\sigma_{N}=\sqrt{0.5 N} \times \sigma_{C R}$. For high CNs we declared states as an interval of CNs (CN21-100, CN101-1000) and modeled their emission distribution as a mixture of normal distributions with weights proportional to the estimated frequencies of each $\mathrm{CN}$. We trained the transition matrix of this HMM with the Baum-Welch algorithm coded in the Pomegranate Python package [88] until convergence. We trained the HMM separately for dogs and wolves, using continuous $\mathrm{CN}$ predictions from read depth for one of the samples as observations (see Table 1). We excluded samples used for training from further analysis.

As samples differ greatly in coverage, which in turn leads to differences in standard deviations in the control regions, we redefined the HMM for each individual separately, so the emission distributions would resemble sample specific standard deviation of the read depth in regions of $C N=2$. The transition matrix is, on the contrary, subspecies specific and does not depend on the sequencing quality. We predict probabilities of each of the declared states at each point by using the forward-reverse algorithm coded in the Pomegranate Python Package. For each individual we thus predict probabilities of each $\mathrm{CN}$ at each 1- 
Kbp window. Individual read depth based predictions of $\mathrm{CN}$ are very noisy and in order to improve them we additionally performed local population-based re-genotyping. For a particular observation of read depth derived raw $\mathrm{CN}$ $c n=x$, we use Bayes' theorem to estimate the probability to draw this value from each of the distributions corresponding to $\mathrm{CN}$ states:

$$
\begin{gathered}
p(C N=N \mid c n \in[x+d x])=\frac{p(c n \in[x+d x] \mid C N=N) \times p(C N=N)}{p(c n \in[x+d x])} \\
=\frac{P D F\left(c n, N, \sqrt{0.5 N} \sigma_{C R}\right) \times d x \times p(N)}{\sum_{c N} P D F\left(c n, N, \sqrt{0.5 N} \sigma_{C R}\right) \times d x}=\frac{P D F\left(c n, N, \sqrt{0.5 N} \sigma_{C R}\right)}{\sum_{c N} P D F\left(c n, N, \sqrt{0.5 N} \sigma_{C R}\right)} \times p(N) ;
\end{gathered}
$$

where $p(N)$ is the expected probability to observe $C N=N$ in the data, the only variable which could be tuned locally. For every 1-Kbp window and each possible state of $C N=$ $N$, we calculated its average probability across all the individuals in 5 consecutive windows, centered at the window of interest, and used this mean probability as a prior for the expected probability $p(N)$ of $C N=N$ in the data.

For a fraction of 1 -Kbp windows $(\sim 2.5 \%$ inside duplications, $\sim 51 \%$ genome wide) we can call the underlying $\mathrm{CN}$ with high confidence $(p>0.99)$ as a unique integer value. But for complex regions of high $\mathrm{CN}$ which are variable across individuals, the probability of each $\mathrm{CN}$ is low $(p<0.99)$. For such windows we consider confidence intervals of the underlying $\mathrm{CN}$. To do so, for each window, we order $\mathrm{CN}$ states according to their probability after population based local re-genotyping, and add them to the interval one by one, until their cumulative probability reaches $p=0.99$ threshold. We further call underlying $\mathrm{CN}$ of the window to belong to this interval. We thus could assess if for a particular window any two individuals have the same $\mathrm{CN}$ (if we confidently call them with the exact value), different (if we confidently call them to belong to non-overlapping $\mathrm{CN}$ intervals), or unresolved (if we call the individuals to belong to overlapping intervals).

We defined duplicated regions as regions of the genome, which harbor at least 5 consecutive windows, which we confidently call as $\mathrm{CN}>=3$ in at least one of the individual canine genomes. The collection of all such regions we call duplication track, and perform all further analyses only for windows which belong to this track.

\section{aCGH data and validation of the method}

For 14 of the samples ( 1 dog, 1 jackal and 11 gray wolves and 1 red wolf) in which we predicted fine scale confidence $\mathrm{CN}$ values, aCGH data assays were available [43] (Table 1). This aCGH chip contains 598,733 probes which target, with a higher density, previously reported regions in the canine genome harboring structural variation [87]. In this study a Boxer sample was used as a reference in the array and we sequenced the same individual in the present study (bxr). Because the aCGH data was based on CanFam2 we generated the 1-Kbps $\mathrm{CN}$ predictions based on this version of the dog genome reference assembly and called confidence $\mathrm{CN}$ intervals for these 14 samples in the described fashion.

We performed quality control of aCGH experiments by assessing density function of aCGH probes for each individual (Additional file 1: Figure S8). The standard deviation for sample ysc was 2.5 times higher than for the rest of the samples, and we thus excluded ysc from subsequent aCGH validation analysis. We than calculated a threshold to separate true aCGH signals corresponding to gains and losses from diploid noise. To do so, we defined true $\mathrm{CN}=2$ windows as the intersection between regions which were previously experimentally identified as diploid $[4,41,42,87]$ and the regions which we confidently called as $\mathrm{CN}=2$ (probability greater than 0.99). As the aCGH chip was designed to target duplications and $\mathrm{CNV}$ regions previously reported in the canine genome, genome-wide 1-Kbp windows may be not covered uniformly with aCGH probes or covered at all, so we restricted our analysis only to the windows which harbor at least 2 different aCGH probes. We plotted the distribution of median aCGH signals for Boxer sample in these subset of windows $(n=1452)$, and used a cutoff for aCGH signal CUTOFF $=a C G H$ $M E A N(C N=2) \pm 3^{*} a C G H_{S D}(C N=2)= \pm 0.20$ to discriminate between true gains and losses from false ones.

To validate our calls, we assessed if the difference in the $\mathrm{CN}$ which we predict computationally is confirmed by aCGH values. For each individual separately, we detected windows inside SDs, which we computationally predicted to be of a different $\mathrm{CN}$ than the reference Boxer sample. This difference could be a duplication compared to Boxer, if the sample $\mathrm{CN}$ is predicted to be higher than in Boxer, or a deletion compared to Boxer, if sample $\mathrm{CN}$ is lower than Boxer's. We assessed the accuracy in detecting duplications and deletions separately, and calculated it as percentage of windows, which we predict to be $\mathrm{CN}$ different from Boxer, which have median aCGH above or below the CUTOFF $= \pm 0.2$ respectively.

\section{Diversity analysis}

Our probabilistic method has enabled us to analyze for the first time the fraction of $\mathrm{CNV}$ genome-wide and compare it to SNP diversity. To avoid sample sizes biases between dogs $(n=11)$ and gray wolves $(n=17)$, we matched the number of individuals from either subspecies by selecting a subset of 11 gray wolves based on various criteria (Additional file 1: Table S3); the selection of samples also ensured that only one gray wolf from each population was used. 


\section{SNP calling and overall SNP diversity}

After mapping sequencing reads to the canine genome with BWA [89], we used the CallableLoci tool of GATK [90], with default parameters, to determine areas of the genome that could be considered callable in each of the samples used in the analysis of CNV. We then defined the "callable genome" as the intersection of the callable regions across all the individuals. In addition, we subtracted from the callable genome the $\mathrm{X}$ chromosome and mitochondrial DNA, those regions that were masked in the version of the dog genome assembly used here (see above) and 1-Kbps windows with $\mathrm{CN}$ exceeding the sample-specific cutoff in at least one sample (Additional file 1: Table S5). After indel realignment we used the UnifiedGenotyper and VariantFiltration tools of GATK [90], with filtering parameters suggested when Variant Quality Score Recalibration (VQSR) is not available [91], to call SNP variants in the total of 11 dogs and 11 gray wolves used in the analysis of the genomic $\mathrm{CNV}$ proportion. For this analysis, however, we only retained those variants within the final callable genome (Additional file 1: Table S5). We then split SNPs into those seen in either dog or gray wolf samples and calculated, as a measure of overall SNP diversity, the number of segregating sites in either subspecies divided by the number of bps in the final callable, allowing for zero or two missing alleles (Additional file 1: Table S5). We also calculated the number of segregating sites per bps of callable using the subset of 8 dogs and 8 gray wolves with raw coverage $>7 \mathrm{X}$. We observed that the callable genome was greatly reduced by including those samples with a lower raw coverage (Additional file 1: Table S5). We therefore also performed the SNP calling and calculated SNP diversity in the subset of 8 dogs and 8 gray wolves with sequencing raw coverage $>7 \mathrm{X}$ (see Table 1). We generated bootstrap values for the observed overall SNP diversity as follows: (i) partition the callable genome into intervals of $1 \mathrm{Mbps}(I)$; (ii) random sampling with replacement of $I$ intervals and re-calculated the number of segregating sites divided by the length of the callable genome.

\section{Genomic fraction of CNVs}

Within dogs and gray wolves separately, we identify $\mathrm{CNV}$ windows as windows for which there are at least two individuals with non-overlapping predicted $\mathrm{CN}$ intervals. We measure variability within subspecies as percentage of variable windows among all the windows inside duplicated regions. In either subspecies we obtained an overall measure of $\mathrm{CN}$ variability as follows: (i) subset 1 -Kbps windows which lie inside duplicated regions of a given subspecies $(N)$; (ii) from those subset 1$\mathrm{Kbp}$ windows which are variable in a given subspecies; (iii) generated bootstrap values by randomly sampling with replacement $N$ windows and re-calculating $\mathrm{CN}$ variability, for a total of 5000 times.
To assess the patterns of variable $\mathrm{CN}$ across different $\mathrm{CN}$ values, we divided all the duplications into the $\mathrm{CN}$ bins. To each $1-\mathrm{Kbp}$ window we assign a value, which is average of median points of $\mathrm{CN}$ intervals across individuals within subspecies. We further created bins of absolute CNs in such a way, that each bin contains at least $5 \%$ of the total number of duplicated windows: low $\mathrm{CN}$ (mean $\mathrm{CN}=2-4$ ), medium $\mathrm{CN}$ (mean $\mathrm{CN}=5-15)$ and high $\mathrm{CN}$ (mean $\mathrm{CN}>15)$. We classified all the windows to the bins and assessed the proportion of variable windows in each of them separately for dogs and wolves. To control for the high levels of noise in individual $\mathrm{CN}$ predictions we assessed variability for regions comprising at least 5 consecutive variable windows. As a separate control, we excluded singletons from the variability calls and required at least 2 individuals to belong to each of the non-overlapping $\mathrm{CN}$ intervals (Fig. 2, Additional file 1: Table S6).

\section{Genes overlapping with genomic duplications and enrichment analysis}

We downloaded the 29,884 Ensembl gene models available for CanFam3.1 from the UCSC Genome Browser [83]. Additionally, we considered as of higher confidence those transcripts, 26,748 genes (89.51\%), comprising at least one exon present in the xenoRef set of positions syntenic to exons in other species $(n=2,381,071)$, which was downloaded from the UCSC Genome Browser [83]. These transcripts were converted back to the gene coordinates and only the total of $N=20,328$ genes in autosomes were considered for further analysis. For the gene enrichment tests we only selected genes which were entirely covered by duplications. We estimated the gene enrichment associated $p$-values by the bootstrap. We performed 10,000 repetitions of shuffling duplications coordinates, while keeping their true size and avoiding placing smaller duplications $(<100 \mathrm{~Kb})$ on gaps in order to generate an empirical distribution of the expected overlap between genes and SDs. The empirical p-value of the true observed value was calculated by dividing the rank of the true observation by the total number of permutations. The enrichment analysis was performed using the elimination algorithm of the TopGO R package [92], which scores GO terms hierarchically and subtracts specific, significant terms from the more global ones to avoid an overrepresentation of the latter. This conditions the results of the recursive tests on the topology of the gene ontology tree and reduces the effect of multiple testing to a level where no further conventional correction is required [93]. Instead, we refined our result set with the browser tool REVIGO [94], which implements semantic search algorithms in order to merge closely related GO terms and extract the most significant relations between them. 


\section{Analysis of CNV differentiation between dogs and gray wolves}

In every 1-Kbps window we used $\mathrm{CN}$ predictions in $\operatorname{dog}$ and gray wolf samples to calculate the $\mathrm{V}_{\mathrm{ST}}$ statistic [51] between the two subspecies. The $\mathrm{V}_{\mathrm{ST}}$ statistic is a variation of the $\mathrm{F}_{\mathrm{ST}}$ [95] to measure between-populations differentiation in CNV regions: $\left(V_{\mathrm{T}}-\mathrm{V}_{\mathrm{S}}\right) / V_{\mathrm{T}}$ where $V_{\mathrm{T}}$ is the variance in the $\mathrm{CN}$ midpoints of all subspecies together, and $V_{S}$ is the weighted average of the variance in $\mathrm{CN}$ midpoints for each subspecies separately. For consistency with the analysis of CNV and SNP diversity we calculated $V_{\text {ST }}$ values between the same subsets of 11 dogs and 11 gray wolves (Additional file 1: Table S3). We looked for genes with median $\mathrm{V}_{\mathrm{ST}}>0.15$ between dogs and wolves, which corresponds to the windows with the top $10 \%$ of $V_{\text {ST }}$ values. We focused on the genes with more than 3 copies in dogs while less than 3 copies in wolves (Additional file 1: Table S9).

\section{Additional file}

Additional file 1: Collection of all supplementary figures and tables. (DOCX $721 \mathrm{~kb})$

\section{Acknowledgements}

We thank Dorina Twigg, Stefan Sirakov, and Jeffrey M. Kidd for their valuable contribution to the analysis and processing of our data.

\section{Funding}

TMB is supported by MINECO BFU2014-55090-P (FEDER), U01 MH106874 grant, Howard Hughes International Early Career, Obra Social "La Caixa" and Secretaria d'Universitats i Recerca del Departament d'Economia i Coneixement de la Generalitat de Catalunya. ISP is supported with a Juan de la Cierva - Formación FJCl-201524,275 fellowship. JHR is supported by the Spanish Ministry of Education under FPI grant (BES-2013-064333). BLG is supported with a Beatriu de Pinós (BP-DGR 2014) fellowship.

\section{Availability of data and materials}

The WGS datasets analysed during the current study are available in the National Center for Biotechnology Information (NCBI) Sequence Read Archive (SRA) database under the accessions SRA068869 (Wang et al. [56]), SRP044399 (Fan et al. [57]) and PRJNA274504 (Freedman et al. [4]) [https:// www.ncbi.nlm.nih.gov/sra/?term=SRA068869, https://www.ncbi.nlm.nih.gov/ sra/?term=SRP044399, https://www.ncbi.nlm.nih.gov/sra/

?term=PRJNA274504].

\section{Authors' contributions}

TMB, BLG, ISP designed the study, analyses and the method; ASA, ISP, JQ performed most of the analyses; MFC, DGS performed variant calling and SNP diversity analysis; OR and JHR performed the experimental analyses; BLG, LFKK contributed to the analyses; OR, GS, AN contributed to the design of the analyses; ISP, JQ, ASA, TMB, BLG wrote the manuscript; AHF, ZF, JN, AB, $\mathrm{CV}$, RW collected samples; all authors read and approved the manuscript.

\section{Ethics approval and consent to participate}

Not applicable.

\section{Consent for publication}

Not applicable.

\section{Competing interests}

The authors declare no conflict of interest. The aCGH dataset analysed during the current study is available in the Gene Expression Omnibus database under the accession GSE58195 (Ramirez et al. [43]) [https:// www.ncbi.nlm.nih.gov/geo/query/acc.cgi?acc=GSE58195].

\section{Publisher's Note}

Springer Nature remains neutral with regard to jurisdictional claims in published maps and institutional affiliations.

\section{Author details}

${ }^{1}$ IBE, Institut de Biologia Evolutiva (Universitat Pompeu Fabra/CSIC), Ciencies Experimentals i de la Salut, 08003 Barcelona, Spain. ${ }^{2}$ CNAG-CRG, Centre for Genomic Regulation (CRG), The Barcelona Institute of Science and Technology (BIST), Barcelona, Spain. ${ }^{3}$ Vetgenomics, 08193 Barcelona, Spain. ${ }^{4}$ Department of Neuroscience, Yale School of Medicine, New Haven, CT, USA. ${ }^{5}$ UCLA, Department of Ecology and Evolutionary Biology, Los Angeles, CA 90095, USA. ${ }^{6}$ Key Laboratory of Bioresources and Ecoenvironment (Ministry of Education), College of Life Sciences, Sichuan University, Chengdu 610064, People's Republic of China. ${ }^{7}$ Institucio Catalana de Recerca i Estudis Avançats (ICREA), 08010 Barcelona, Catalonia, Spain. ${ }^{8}$ Cornell University, Department of Biological Statistics and Computational Biology, New York, NY 14853, USA. ${ }^{9}$ Estación Biológica de Doñana EBD-CSIC, Department of Integrative Ecology, 41092 Sevilla, Spain.

Received: 10 April 2017 Accepted: 17 November 2017

Published online: 19 December 2017

\section{References}

1. Vilà C, Savolainen P E. Maldonado J R. Amorim I E. Rice J L. Honeycutt R, et al. Multiple and Ancient Origins of the Domestic Dog. 1997. Science. doi:10.1126/science.276.5319.1687.

2. Lindblad-Toh K. Genome sequence, comparative analysis and haplotype structure of the domestic dog. Nature. 2005;438(7069):803-19.

3. Germonpré M, Sablin MV, Stevens RE, Hedges REM, Hofreiter M, Stiller M, et al. Fossil dogs and wolves from Palaeolithic sites in Belgium, the Ukraine and Russia: osteometry, ancient DNA and stable isotopes. J Archaeol Sci. 2009;36:473-90.

4. Freedman AH, Gronau I, Schweizer RM, Ortega-Del Vecchyo D, Han EE, Silva PM, et al. Genome sequencing highlights the dynamic early history of dogs. PLoS Genet. 2014;10:e1004016. doi:10.1371/journal.pgen.1004016.

5. Boyko AR. The domestic dog: man's best friend in the genomic era. Genome Biol. 2011;12:216. doi:10.1186/gb-2011-12-2-216.

6. Larson G, Bradley DG. How much is that in dog years? The advent of canine population genomics. PLoS Genet. 2014;10:e1004093. doi:10.1371/journal. pgen.1004093.

7. Skoglund P. Estimation of population divergence times from nonoverlapping genomic sequences: examples from dogs and wolves. Mol Biol Evol. 2011;28(4):1505-17.

8. Thalmann O, Shapiro B, Cui P, Schuenemann VJ, Sawyer SK, Greenfield DL, et al. Complete mitochondrial genomes of ancient Canids suggest a European origin of domestic dogs. Science (80- ). 2013;2013:342. http:// science.sciencemag.org/content/342/6160/871.full. Accessed 14 Sep 2016

9. Frantz LAF, Mullin VE, Pionnier-Capitan M, Lebrasseur O, Ollivier M, Perri A, et al. Genomic and archaeological evidence suggest a dual origin of domestic dogs. Science (80- ). 2016;2016:352.

10. Wang L, Ma Y-P, Zhou Q-J, Zhang Y-P, Savolaimen P, Wang G-D. The geographical distribution of grey wolves (Canis Lupus) in China: a systematic review. Zool Res. 2016;37:315-26. 10.13918/j.issn.2095-8137.2016.6.315.

11. Vaysse A, Ratnakumar A, Derrien T, Axelsson E, Rosengren Pielberg G, Sigurdsson $\mathrm{S}$, et al. Identification of genomic regions associated with phenotypic variation between dog breeds using selection mapping. PLoS Genet. 2011;7:e1002316. doi:10.1371/journal.pgen.1002316.

12. Irion DN, Schaffer AL, Famula TR, Eggleston ML, Hughes SS, Pedersen NC. Analysis of genetic variation in 28 dog breed populations with 100 microsatellite markers. J Hered. 2003;94:81-7. doi:10.1093/JHERED/ESG004.

13. Ostrander EA, Wayne RK. The canine genome. Genome Res. 2005;15:170616. doi:10.1101/gr.3736605.

14. Gundry RL, Allard MW, Moretti TR, Honeycutt RL, Wilson MR, Monson $\mathrm{KL}$, et al. Mitochondrial DNA analysis of the domestic dog: control region variation within and among breeds. J Forensic Sci. 2007:52:56272. doi:10.1111/j.1556-4029.2007.00425.x.

15. Shannon LM, Boyko RH, Castelhano M, Corey E, Hayward JJ, McLean $C$, et al. Genetic structure in village dogs reveals a central Asian 
domestication origin. Proc Natl Acad Sci U S A. 2015;112:13639-44. doi:10.1073/pnas.1516215112.

16. Decker B, Davis BW, Rimbault M, Long AH, Karlins E, Jagannathan V, et al. Comparison against 186 canid whole-genome sequences reveals survival strategies of an ancient clonally transmissible canine tumor. Genome Res. 2015;25:1646-55. doi:10.1101/gr.190314.115.

17. Gray MM, Granka JM, Bustamante CD, Sutter NB, Boyko AR, Zhu L, et al. Linkage disequilibrium and demographic history of wild and domestic Canids. Genetics. 2009;181:1493-505. doi:10.1534/genetics.108.098830.

18. Pang J-F, Kluetsch C, Zou X-J, Zhang A-B, Luo L-Y, Angleby H, et al. mtDNA data indicate a single origin for dogs south of Yangtze River, less than 16,300 years ago, from numerous wolves. Mol Biol Evol. 2009;26:2849-64. doi:10.1093/molbev/msp195.

19. Boyko AR, Quignon P, Li L, Schoenebeck JJ, Degenhardt JD, Lohmueller KE, et al. A simple genetic architecture underlies morphological variation in dogs. PLOS Biol. 2010;8:e1000451. doi:10.1371/journal.pbio.1000451.

20. VonHoldt BM, Pollinger JP, Lohmueller KE, Han E, Parker HG, Quignon P, et al. Genome-wide SNP and haplotype analyses reveal a rich history underlying dog domestication. Nature. 2010:464:898-902. doi:10.1038/nature08837.

21. Karlsson EK, Baranowska I, Wade CM, Salmon Hillbertz NHC, Zody MC, Anderson $\mathrm{N}$, et al. Efficient mapping of mendelian traits in dogs through genome-wide association. Nat Genet. 2007;39:1321-8. doi:10.1038/ng.2007.10.

22. Auton A, Rui Li Y, Kidd J, Oliveira K, Nadel J, Holloway JK, et al. Genetic recombination is targeted towards gene promoter regions in dogs. PLoS Genet. 2013;9:1-2.

23. Axelsson E, Ratnakumar A, Arendt M-L, Magbool K, Webster MT, Perloski M, et al. The genomic signature of dog domestication reveals adaptation to a starch-rich diet. Nature. 2013;495:360-4. doi:10.1038/nature11837.

24. Bannasch D, Young A, Myers J, Truvé K, Dickinson P, Gregg J, et al. Localization of canine Brachycephaly using an across breed mapping approach. PLoS One. 2010;5:e9632. doi:10.1371/journal.pone.0009632.

25. Cadieu E, Neff MW, Quignon P, Walsh K, Chase K, Parker HG, et al. Coat variation in the domestic dog is governed by variants in three genes. Science. 2009;326:150-3. doi:10.1126/science.1177808.

26. Olsson M, Meadows JRS, Truvé K, Rosengren Pielberg G, Puppo F, Mauceli E, et al. A novel unstable duplication upstream of HAS2 predisposes to a breeddefining skin phenotype and a periodic fever syndrome in Chinese Shar-Pei dogs. PLoS Genet. 2011;7:e1001332. doi:10.1371/journal.pgen.1001332.

27. Quilez J, Short AD, Martínez V, Kennedy LJ, Ollier W, Sanchez A, et al. A selective sweep of \&gt;8 Mb on chromosome 26 in the boxer genome. BMC Genomics. 2011;12:339. doi:10.1186/1471-2164-12-339.

28. Salmon Hillbertz NHC, Isaksson M, Karlsson EK, Hellmén E, Pielberg GR, Savolainen P, et al. Duplication of FGF3, FGF4, FGF19 and ORAOV1 causes hair ridge and predisposition to dermoid sinus in ridgeback dogs. Nat Genet. 2007;39:1318-20. doi:10.1038/ng.2007.4

29. Schoenebeck JJ, Hutchinson SA, Byers A, Beale HC, Carrington B, Faden DL, et al. Variation of BMP3 contributes to dog breed skull diversity. PLoS Genet. 2012;8:e1002849. doi:10.1371/journal.pgen.1002849.

30. Berglund J, Nevalainen EM, Molin A-M, Perloski M, André C, Zody MCM, et al. Novel origins of copy number variation in the dog genome. Genome Biol. 2012;13:R73. doi:10.1186/gb-2012-13-8-r73.

31. Coe BP, Witherspoon K, Rosenfeld JA, van Bon BWM, Vulto-van Silfhout AT, Bosco $P$, et al. Refining analyses of copy number variation identifies specific genes associated with developmental delay. Nat Genet. 2014;46:1063-71. doi:10.1038/ng.3092.

32. Fanciulli M, Norsworthy PJ, Petretto E, Dong R, Harper L, Kamesh L, et al. FCGR3B copy number variation is associated with susceptibility to systemic, but not organ-specific, autoimmunity. Nat Genet. 2007;39:721-3. doi:10.1038/ng2046.

33. Alkan C, Kidd JM, Marques-Bonet T, Aksay G, Antonacci F, Hormozdiari F, et al. Personalized copy number and segmental duplication maps using next-generation sequencing. Nat Genet. 2009;41:1061-7. doi:10.1038/ng.437.

34. Ghosh S, Qu Z, Das PJ, Fang E, Juras R, Cothran EG, et al. Copy number variation in the horse genome. PLoS Genet. 2014;10:e1004712. doi:10.1371/ journal.pgen.1004712.

35. Chain FJJ, Feulner PGD, Panchal M, Eizaguirre C, Samonte IE, Kalbe M, et al. Extensive copy-number variation of young genes across stickleback populations. PLoS Genet. 2014;10:e1004830. doi:10.1371/journal.pgen.1004830.

36. Yi G, Qu L, Liu J, Yan Y, Xu G, Yang N. Genome-wide patterns of copy number variation in the diversified chicken genomes using next-generation sequencing. BMC Genomics. 2014;15:962. doi:10.1186/1471-2164-15-962.
37. Jiang J, Wang J, Wang H, Zhang Y, Kang H, Feng X, et al. Global copy number analyses by next generation sequencing provide insight into pig genome variation. BMC Genomics. 2014;15:593. doi:10.1186/1471-2164-15-593.

38. Sudmant PH, Kitzman JO, Antonacci F, Alkan C, Malig M, Tsalenko A, et al. Diversity of human copy number variation and multicopy genes. Science (80- ). 2010;330:641-6. doi:10.1126/science.1197005.

39. Sudmant PH, Huddleston J, Catacchio CR, Malig M, Hillier LW, Baker C, et al. Evolution and diversity of copy number variation in the great ape lineage. Genome Res. 2013;23:1373-82. doi:10.1101/gr.158543.113.

40. Sudmant PH, Mallick S, Nelson BJ, Hormozdiari F, Krumm N, Huddleston J, et al. Global diversity, population stratification, and selection of human copy-number variation. Science (80- ). 2015;349: aab3761. doi:10.1126/science.aab3761.

41. Chen W-K, Swartz JD, Rush LJ, Alvarez CE. Mapping DNA structural variation in dogs. Genome Res. 2008;19:500-9. doi:10.1101/gr.083741.108.

42. Nicholas TJ, Cheng Z, Ventura M, Mealey K, Eichler EE, Akey JM. The genomic architecture of segmental duplications and associated copy number variants in dogs. Genome Res. 2009;19:491-9. doi:10.1101/gr.084715.108.

43. Ramirez O, Olalde I, Berglund J, Lorente-Galdos B, HernandezRodriguez J, Quilez J, et al. Analysis of structural diversity in wolf-like canids reveals post-domestication variants. BMC Genomics. 2014;15: 465. doi:10.1186/1471-2164-15-465.

44. Poorman K, Borst L, Moroff S, Roy S, Labelle P, Motsinger-Reif A, et al. Comparative cytogenetic characterization of primary canine melanocytic lesions using array CGH and fluorescence in situ hybridization. Chromosom Res. 2015;23:171-86. doi:10.1007/s10577-014-9444-6.

45. Rossi E, Radi O, De Lorenzi L, Vetro A, Groppetti D, Bigliardi E, et al. Sox9 duplications are a relevant cause of Sry-negative XX sex reversal dogs. PLoS One. 2014;9:e101244. doi:10.1371/journal.pone.0101244.

46. Molin A-M, Berglund J, Webster MT, Lindblad-Toh K. Genome-wide copy number variant discovery in dogs using the CanineHD genotyping array. BMC Genomics. 2014;15:210. doi:10.1186/1471-2164-15-210.

47. Coe BP, Ylstra B, Carvalho B, Meijer GA, MacAulay C, Lam WL. Resolving the resolution of array CGH. Genomics. 2007;89:647-53.

48. Sharp AJ, Itsara A, Cheng Z, Alkan C, Schwartz S, Eichler EE. Optimal design of oligonucleotide microarrays for measurement of DNA copy-number. Hum Mol Genet. 2007;16:2770-9. doi:10.1093/hmg/ddm234.

49. Cooper GM, Zerr T, Kidd JM, Eichler EE, Nickerson DA. Systematic assessment of copy number variant detection via genome-wide SNP genotyping. Nat Genet. 2008:40:1199-203. doi:10.1038/ng.236.

50. Winchester L, Yau C, Ragoussis J. Comparing CNV detection methods for SNP arrays. Brief Funct Genomic Proteomic. 2009;8:353-66. doi:10.1093/bfgp/elp017.

51. Redon R, Ishikawa S, Fitch KR, Feuk L, Perry GH, Andrews TD, et al. Global variation in copy number in the human genome. Nature. 2006;444:444-54. doi:10.1038/nature05329.

52. Lou H, Li S, Yang Y, Kang L, Zhang $X$, Jin W, et al. A map of copy number variations in Chinese populations. PLoS One. 2011;6:e27341. doi:10.1371/ journal.pone.0027341.

53. Zhao M, Wang Q, Wang Q, Jia P, Zhao Z. Computational tools for copy number variation (CNV) detection using next-generation sequencing data: features and perspectives. BMC Bioinformatics. 2013;14(Suppl 11):S1. doi:10. 1186/1471-2105-14-S11-S1.

54. Girirajan $\mathrm{S}$, Campbell CD, Eichler EE. Human copy number variation and complex genetic disease. Annu Rev Genet. 2011;45:203-26. doi:10.1146/ annurev-genet-102209-163544.

55. Yang Y, Chung EK, YL W, Savelli SL, Nagaraja HN, Zhou B, et al. Gene copynumber variation and associated polymorphisms of complement component C4 in human systemic lupus erythematosus (SLE): low copy number is a risk factor for and high copy number is a protective factor against SLE susceptibility in European America. Am J Hum Genet. 2007;80:1037-54.

56. Wang G, Zhai W, Yang H, Fan R, Cao X, Zhong L, et al. The genomics of selection in dogs and the parallel evolution between dogs and humans. Nat Commun. 2013;4:1860. doi:10.1038/ncomms2814.

57. Fan Z, Silva P, Gronau I, Wang S, Armero AS, Schweizer RM, et al. Worldwide patterns of genomic variation and admixture in gray wolves. Genome Res. 2016;26:163-73. doi:10.1101/gr.197517.115.

58. Kidd JM, Cooper GM, Donahue WF, Hayden HS, Sampas N, Graves T, et al. Mapping and sequencing of structural variation from eight human genomes. Nature. 2008;453:56-64. doi:10.1038/nature06862.

59. Armengol L, Villatoro S, González JR, Pantano L, García-Aragonés M, Rabionet $R$, et al. Identification of copy number variants defining 
genomic differences among major human groups. PLoS One. 2009;4 e7230. doi:10.1371/journal.pone.0007230.

60. Marques-Bonet T, Kidd JM, Ventura M, Graves TA, Cheng Z, Hillier LW, et al A burst of segmental duplications in the genome of the African great ape ancestor. Nature. 2009;457:877-81. doi:10.1038/nature07744.

61. Barclay AN, Brown MH. The SIRP family of receptors and immune regulation. Nat Rev Immunol. 2006;6:457-64. doi:10.1038/nri1859.

62. Pirooznia M, Goes FS, Zandi PP. Whole-genome CNV analysis: advances in computational approaches. Front Genet. 2015;6:138. doi:10.3389/fgene.2015.00138.

63. Larson G, Karlsson EK, Perri A, Webster MT, Ho SYW, Peters J, et al. Rethinking dog domestication by integrating genetics, archeology, and biogeography. Proc Natl Acad Sci U S A. 2012;109:8878-83. doi:10.1073/ pnas.1203005109.

64. Cruz F, Vila C, Webster MT. The legacy of domestication: accumulation of deleterious mutations in the dog genome. Mol Biol Evol. 2008;25:2331-6. doi:10.1093/molbev/msn177.

65. Marsden CD, Ortega-Del Vecchyo D, O'Brien DP, Taylor JF, Ramirez O, Vilà C, et al. Bottlenecks and selective sweeps during domestication have increased deleterious genetic variation in dogs. Proc Natl Acad Sci U S A. 2016;113:152-7. doi:10.1073/pnas.1512501113.

66. Carvalho CMB, Lupski JR. Mechanisms underlying structural variant formation in genomic disorders. Nat Rev Genet. 2016;17:224-38. doi:10. 1038/nrg.2015.25.

67. Miller DE, Hawley RS. Tetrad analysis in the mouse. Nat Genet. 2014;46: 1045-6. doi:10.1038/ng.3104.

68. Freedman AH, Schweizer RM, Ortega-Del Vecchyo D, Han E, Davis BW, Gronau I, et al. Demographically-based evaluation of genomic regions under selection in domestic dogs. PLoS Genet. 2016;12:e1005851. doi:10. 1371/journal.pgen.1005851.

69. Allendorf FW. Genetic drift and the loss of alleles versus heterozygosity. Zoo Biol. 1986;5:181-90. doi:10.1002/zoo.1430050212.

70. Maruyama T, Fuerstt PA. POPULATION BOTTLENECKS AND NONEQUILIBRIUM MODELS IN POPULATION GENETICS. 11. NUMBER O F ALLELES IN a SMALL POPULATION T H a T WAS FORMED BY a RECENT BOTTLENECK HE fate of genes in a population that experiences a sudden reduction in. Genetics. 1985;111:675-89. https://www.ncbi.nlm.nih.gov/pmc/ articles/PMC1202664/pdf/675.pdf. Accessed 24 Mar 2017.

71. Hodgkinson A, Eyre-Walker A. Human triallelic sites: evidence for a new mutational mechanism? Genetics. 2010;184:233-41. doi:10.1534/genetics. 109.110510.

72. Handsaker RE, Van Doren V, Berman JR, Genovese G, Kashin S, Boettger LM, et al. Large multiallelic copy number variations in humans. Nat Genet. 2015; 47:296-303. doi:10.1038/ng.3200.

73. Duvaux L, Geissmann Q, Gharbi K, Zhou J-J, Ferrari J, Smadja CM, et al. Dynamics of copy number variation in host races of the pea aphid. Mol Biol Evol. 2015;32:63-80.

74. Skjeldal $\mathrm{OH}$, Stokke O, Refsum S, Norseth J, Petit $\mathrm{H}$. Clinical and biochemical heterogeneity in conditions with phytanic acid accumulation. J Neurol Sci. 1987;77:87-96. http://www.ncbi.nlm.nih.gov/ pubmed/2433405. Accessed 4 Oct 2017

75. Skarnes WC, Rosen B, West AP, Koutsourakis M, Bushell W, lyer V, et al. A conditional knockout resource for the genome-wide study of mouse gene function. Nature. 2011:474:337-42. doi:10.1038/nature10163.

76. Olsson M, Frankowiack M, Tengvall K, Roosje P, Fall T, Ivansson E, et al. The dog as a genetic model for immunoglobulin a (lgA) deficiency: identification of several breeds with low serum IgA concentrations. Vet Immunol Immunopathol. 2014;160:255-9. doi:10.1016/j.vetimm.2014.05.010.

77. Karyadi DM, Karlins E, Decker B, vonHoldt BM, Carpintero-Ramirez G, Parker $\mathrm{HG}$, et al. A copy number variant at the KITLG locus likely confers risk for canine Squamous cell carcinoma of the digit. PLoS Genet. 2013;9:e1003409. doi:10.1371/journal.pgen.1003409

78. Lao O, de Gruijter JM, van Duijn K, Navarro A, Kayser M. Signatures of positive selection in genes associated with human skin pigmentation as revealed from analyses of single nucleotide polymorphisms. Ann Hum Genet. 2007;71:354-69. doi:10.1111/j.1469-1809.2006.00341.x.

79. Metzger J, Karwath M, Tonda R, Beltran S, Águeda L, Gut M, et al. Runs of homozygosity reveal signatures of positive selection for reproduction traits in breed and non-breed horses. BMC Genomics. 2015;16:764. doi:10.1186/ s12864-015-1977-3.

80. Gutierrez-Gil B, Arranz JJ, Wiener P. An interpretive review of selective sweep studies in Bos Taurus cattle populations: identification of unique and shared selection signals across breeds. Front Genet. 2015;6:167. doi:10.3389/ fgene.2015.00167.

81. Miller CT, Beleza S, Pollen AA, Schluter D, Kittles RA, Shriver MD, et al. Cisregulatory changes in kit Ligand expression and parallel evolution of pigmentation in sticklebacks and humans. Cell. 2007;131:1179-89. doi:10. 1016/j.cell.2007.10.055.

82. Mengel-From J, Wong TH, Morling N, Rees JL, Jackson IJ. Genetic determinants of hair and eye colours in the Scottish and Danish populations. BMC Genet. 2009;10:88. doi:10.1186/1471-2156-10-88.

83. Raney BJ, Dreszer TR, Barber GP, Clawson H, Fujita PA, Wang T, et al. Track data hubs enable visualization of user-defined genome-wide annotations on the UCSC genome browser. Bioinformatics. 2014;30:1003-5. doi:10.1093/ bioinformatics/btt637.

84. Smit A. The origin of interspersed repeats in the human genome Arian FA Smit. Curr Opin Genet Dev. 1996:6:743-8.

85. Benson G. Tandem repeats finder: a program to analyze DNA sequences. Nucleic Acids Res. 1999;27:573-80. http://www.ncbi.nlm.nih.gov/pubmed/ 9862982. Accessed 24 Feb 2017.

86. Hach F, Hormozdiari F, Alkan C, Hormozdiari F, Birol I, Eichler EE, et al. mrsFAST: a cache-oblivious algorithm for short-read mapping. Nat Methods. 2010;7:576-7. doi:10.1038/nmeth0810-576

87. Nicholas TJ, Baker C, Eichler EE, Akey JM. A high-resolution integrated map of copy number polymorphisms within and between breeds of the modern domesticated dog. BMC Genomics. 2011:12:414 doi:10.1186/1471-2164-12-414.

88. Schreiber J. Pomegranate. 2014. https://github.com/jmschrei/pomegranate.

89. Li H, Durbin R. Fast and accurate short read alignment with burrows-wheeler transform. Bioinformatics. 2009;25:1754-60. doi:10.1093/bioinformatics/btp324.

90. DePristo MA, Banks E, Poplin R, Garimella KV, Maguire JR, Hartl C, et al. A framework for variation discovery and genotyping using next-generation DNA sequencing data. Nat Genet. 2011;43:491-8. doi:10.1038/ng.806.

91. Van der Auwera GA, Carneiro MO, Hartl C, Poplin R, Del Angel G, LevyMoonshine A, et al. From FastQ data to high confidence variant calls: the genome analysis toolkit best practices pipeline. Curr Protoc Bioinforma. 2013;43:11.10.1-33. doi:10.1002/0471250953.bi1110s43.

92. Alexa A. Rahnenfuhrer J. TopGO. 2016; https://bioconductor.org/packages/ release/bioc/html/topGO.html.

93. Alexa A, Joorg Rahnenfuhrer. TopGO Manual, page 19, section 6.2. 2017. https:// bioconductor.org/packages/release/bioc/vignettes/topGO/inst/doc/topGO.pdf.

94. Supek F, Bošnjak M, Škunca N, Šmuc T. REVIGO summarizes and visualizes long lists of gene ontology terms. PLoS One. 2011;6:e21800. doi:10.1371/ journal.pone.0021800.

95. Weir BS, Cockerham CC. Estimating F-statistics for the analysis of population structure. Evolution (N Y). 1984;38:1358. doi:10.2307/2408641.

\section{Submit your next manuscript to BioMed Central and we will help you at every step:}

- We accept pre-submission inquiries

- Our selector tool helps you to find the most relevant journal

- We provide round the clock customer support

- Convenient online submission

- Thorough peer review

- Inclusion in PubMed and all major indexing services

- Maximum visibility for your research

Submit your manuscript at www.biomedcentral.com/submit 